Proceedings Book of International Work shop 1 (2016)) Jun Turky 1 (2017) Elif Özkara Canfes, Joeri Van der Veken and Cornelia-Livia Bejan

Recieved: January 29,2017

Accepted: July 17, 2017

DOI: 10.24064/iwts2016.2017.11

\title{
On Slant Curves with Pseudo-Hermitian $C$-parallel Mean Curvature Vector Fields
}

\author{
Cihan Özgür \\ Cihan Özgür: Balıkesir University, Department of Mathematics, 10145, Çă̆ış, Balıkesir, \\ Turkey, e-mail: cozgur@balikesir.edu.tr
}

\begin{abstract}
We study pseudo-Hermitian $C$-parallel and $C$-proper slant curves in contact metric 3-manifolds. As an application, we give two examples of pseudo-Hermitian Legendre circle and pseudo-Hermitian slant helix in Sasakian Heisenberg group.
\end{abstract}

Keywords. C-parallel mean curvature vector · slant curve · Heisenberg group.

MSC 2010 Classification. Primary: 53C25; Secondary:53A05 · 53C40.

\section{$1 \quad$ INTRODUCTION}

In [7], Chen defined biharmonic submanifold as a Riemannian submanifold with vanishing Laplacian of mean curvature vector field $\Delta H$. Curves in a Euclidean space satisfying the condition $\Delta^{\perp} H=\lambda H$ were classified in [2], by Barros and Garay, where $\Delta^{\perp}$ denotes the Laplacian of the curve in the normal bundle and $\lambda$ is a real valued function. In the real space form, the classification of curves satisfying $\Delta H=\lambda H$ and $\Delta^{\perp} H=\lambda H$ were given in [1], by Arroyo, Barros and Garay.

A curve in a contact metric manifold is said to be slant [9], if its tangent vector field has a constant angle with the Reeb vector field. In particular, if the contact angle is equal to $\frac{\pi}{2}$, then the curve is called a Legendre curve. In [8], Cho and Lee studied slant curves in pseudo-Hermitian contact 3-manifolds. Legendre curves with pseudo-Hermitian parallel mean curvature vector field, pseudo-Hermitian proper mean curvature vector field and pseudoHermitian proper mean curvature vector field in the normal bundle in contact pseudo-Hermitian 3-manifolds were studied by Lee in [12]. In [14], the present author and Güvenç studied slant curves with pseudo-Hermitian parallel mean curvature vector field, pseudo-Hermitian proper mean curvature vector field and pseudo-Hermitian proper mean curvature vector field in the normal bundle in contact pseudo-Hermitian 3-manifolds. The notions of $C$-parallel and $C$-proper 
curves in the tangent and normal bundles were introduced by Lee, Suh and Lee in [13]. A curve in an almost contact metric manifold is defined to be $C$-parallel if $\nabla_{T} H=\lambda \xi, C$-proper if $\Delta H=\lambda \xi, C$-parallel in the normal bundle if $\nabla_{T}^{\perp} H=\lambda \xi, C$-proper in the normal bundle if $\Delta^{\perp} H=\lambda \xi$, where $T$ is the unit tangent vector field of the curve and $\lambda$ is a differentiable function along the curve. In [13], Lee, Suh and Lee studied $C$-parallel and $C$-proper slant curves in Sasakian 3-manifolds. $C$-parallel and $C$-proper slant curves in trans-Sasakian manifolds were studied in [15], by Güvenç and the present author. On the other hand, slant and Legendre curves in Bianchi-Cartan-Vranceanu geometry were studied by Călin and Crasmareanu in [6]. Slant curves in normal almost contact geometry were studied in [5].

Motivated by the above studies, in the present paper, we study pseudoHermitian $C$-parallel and $C$-proper slant curves in contact metric 3 -manifolds. We give two examples of pseudo-Hermitian Legendre circle and pseudo-Hermitian slant helix in Sasakian Heisenberg group.

\section{$2 \quad$ PRELIMINARIES}

Let $M$ be a $(2 n+1)$-dimensional manifold. $M$ is called a contact manifold [3] if there exists a global 1 -form $\eta$ such that $\eta \wedge(d \eta)^{n} \neq 0$ everywhere on $M$. Given a contact form $\eta$, there exists a unique vector field $\xi$, the characteristic vector field, which satisfies $\eta(\xi)=1$ and $d \eta(X, \xi)=0$ for any vector field $X$ on $M$. There exists an associated Riemannian metric $g$ and a $(1,1)$-type tensor field $\varphi$ satisfying

$$
\varphi^{2} X=-X+\eta(X) \xi, \quad \eta(X)=g(X, \xi), \quad d \eta(X, Y)=g(X, \varphi Y),
$$

for all $X, Y \in \chi(M)$. From (2.1), it is easy to see that

$$
\varphi \xi=0, \quad \eta \circ \varphi=0, \quad g(\varphi X, \varphi Y)=g(X, Y)-\eta(X) \eta(Y) .
$$

A Riemannian manifold equipped with the structure tensors $(\varphi, \xi, \eta, g)$ satisfying (2.1) is called a contact metric manifold. It is denoted by $M=\{M, \varphi, \xi, \eta, g\}$. The operator $h$ is defined by $h=\frac{1}{2} L_{\xi} \varphi$, where $L_{\xi}$ is the Lie differentiation operator in the characteristic direction $\xi$. From the definition of $h$, it is easy to see that $h$ is symmetric and satisfies the following equations (see [3], page 67):

$$
h \xi=0, \quad h \varphi=-\varphi h, \quad \nabla_{X} \xi=-\varphi X-\varphi h X,
$$

where $\nabla$ denotes the Levi-Civita connection.

For a $(2 n+1)$-dimensional contact metric manifold $M=\{M, \varphi, \xi, \eta, g\}$, the almost complex structure $J$ on $M \times \mathbb{R}$ is defined by

$$
J\left(X, f \frac{d}{d t}\right)=\left(\varphi X-f \xi, \eta(X) \frac{d}{d t}\right),
$$

where $X$ is a vector field tangent to $M, t$ is the coordinate function of $\mathbb{R}$ and $f$ is a $C^{\infty}$ function on $M \times \mathbb{R}$. If $J$ is integrable then the contact metric manifold $M$ is called a Sasakian manifold [3]. 
For a $(2 n+1)$-dimensional contact metric manifold $M=\{M, \varphi, \xi, \eta, g\}$ provides a splitting of the tangent bundle

$$
T M=\operatorname{Ker}(\varphi) \oplus \operatorname{Im}(\varphi)
$$

and the restriction $J=\left.\varphi\right|_{D}$ defines an almost complex structure on $D=\operatorname{Im}(\varphi)$. There is a well-known concept of almost $C R$-structure as follows: Let $M$ be a $(2 n+s)$-dimensional smooth manifold. Let $\mathcal{D}$ be a smooth distribution on $M$ of real dimension $2 n$ and $J$ a $(1,1)$-tensor field on $M$ such that

$$
J^{2} X=-X, \quad X \in \mathcal{D} .
$$

Then $(\mathcal{D}, J)$ is called almost complex distribution (or an almost $C R$-structure). Then $M$ is an almost $C R$-manifold (or a contact strongly pseudo-convex pseudoHermitian manifold) [3].

The Tanaka-Webster connection $\hat{\nabla}$ (or the pseudo-Hermitian connection) ([16], [18]) on a contact strongly pseudo-convex pseudo-Hermitian manifold $M$ is defined by

$$
\widehat{\nabla}_{X} Y=\nabla_{X} Y+\eta(X) \varphi Y+\left(\nabla_{X} \eta\right)(Y) \xi-\eta(Y) \nabla_{X} \xi
$$

for all $X, Y \in \chi(M)$. By the use of (2.3), $\widehat{\nabla}$ can be rewritten as

$$
\hat{\nabla}_{X} Y=\nabla_{X} Y+\eta(X) \varphi Y+\eta(Y)(\varphi X+\varphi h X)-g(\varphi X+\varphi h X, Y) \xi .
$$

From (2.5), the torsion of the Tanaka-Webster connection $\widehat{\nabla}$ is

$$
\widehat{T}(X, Y)=2 g(X, \varphi Y) \xi+\eta(Y) \varphi h X-\eta(X) \varphi h Y .
$$

If $M$ is a Sasakian manifold, since $h=0$, then the equations (2.5) and (2.6) turn into

$$
\begin{gathered}
\hat{\nabla}_{X} Y=\nabla_{X} Y+\eta(X) \varphi Y+\eta(Y) \varphi X-g(\varphi X, Y) \xi \\
\widehat{T}(X, Y)=2 g(X, \varphi Y) \xi
\end{gathered}
$$

respectively.

\section{Slant CURVES IN CONTACT PSEUDO-HERMITIAN GEOMETRY}

Let $M=\{M, \varphi, \xi, \eta, g\}$ be a contact metric 3-manifold and $\gamma: I \rightarrow M$ a curve parametrized by arc-length in $M$. The Frenet frame field $\{T, N, B\}$ along $\gamma$ for the pseudo-Hermitian connection $\hat{\nabla}$ can be defined by

$$
\begin{aligned}
& \widehat{\nabla}_{T} T=\widehat{\kappa} N, \\
& \widehat{\nabla}_{T} N=-\widehat{\kappa} T+\widehat{\tau} B, \\
& \widehat{\nabla}_{T} B=-\widehat{\tau} N,
\end{aligned}
$$


where $\widehat{\kappa}=\left\|\widehat{\nabla}_{T} T\right\|$ is the pseudo-Hermitian curvature of $\gamma$ and $\widehat{\tau}$ its pseudoHermitian torsion [8]. Similar to the general curve theory, a curve, whose pseudo-Hermitian curvature and pseudo-Hermitian torsion are non-zero constants, is called a pseudo-Hermitian helix. Curves with constant non-zero pseudoHermitian curvature and zero pseudo-Hermitian torsion are called pseudo-Hermitian circles. Pseudo-Hermitian geodesics are curves whose pseudo-Hermitian curvature and pseudo-Hermitian torsion are zero [8].

Let $\gamma: I \rightarrow M$ be a Frenet curve parametrized by arc-length parameter $s$ in a contact metric 3-manifold $M$. The contact angle $\alpha(s)$ is a function defined by $\cos [\alpha(s)]=g(T(s), \xi)$. If the contact angle $\alpha(s)$ is a constant, then $\gamma$ is called a slant curve [9]. Slant curves with contact angle $\pi / 2$ are traditionally called Legendre curves [3].

Throughout the present paper, we assume that all curves are non-geodesic Frenet curves, that is, $\widehat{\kappa} \neq 0$.

In [8], Cho and Lee proved the following three propositions:

Proposition 3.1. [8] A curve $\gamma$ for $\widehat{\nabla}$ is a slant curve if and only if it satisfies $\eta(N)=0$.

Proposition 3.2. [8] Let $\gamma$ be a slant curve for $\widehat{\nabla}$ in a 3-dimensional contact metric manifold $M$. Then the ratio of $\widehat{\tau}$ and $\widehat{\kappa}$ is a constant.

Note that

$$
\frac{\widehat{\tau}}{\widehat{\kappa}}=\cot \alpha_{0},
$$

where $\alpha_{0}$ is the contact angle of $\gamma[14]$.

Proposition 3.3. [8] If a curve in a 3-dimensional contact metric manifold for Tanaka-Webster connection $\widehat{\nabla}$ is a Legendre curve, then $\widehat{\tau}=0$.

In [14], the present author and Güvenç showed that the converse statement of the above proposition is also true. They gave the following result:

Corollary 3.4. [14] Let $\gamma$ be a slant curve for Tanaka-Webster connection $\hat{\nabla}$ with contact angle $\alpha_{0}$ in a 3-dimensional contact metric manifold $M$. Then $\gamma$ is a Legendre curve if and only if $\widehat{\tau}=0$.

The pseudo-Hermitian mean curvature vector field $\widehat{H}$ of a curve $\gamma$ in a 3dimensional contact metric manifold is defined by

$$
\widehat{H}=\widehat{\nabla}_{T} T=\widehat{\kappa} N,
$$

(see [12]). From (4.1), it is easy to see that

$$
\widehat{\nabla}_{T} \widehat{H}=-\widehat{\kappa}^{2} T+\widehat{\kappa}^{\prime} N+\widehat{\kappa} \widehat{\tau} B
$$




$$
\widehat{\nabla} \frac{\perp}{T} \widehat{H}=\widehat{\kappa}^{\prime} N+\widehat{\kappa} \widehat{\tau} B
$$

where $\widehat{H}$ is the pseudo-Hermitian mean curvature vector field of $\gamma$ [12].

Definition 4.1. Let $H$ be the mean curvature vector field of a curve $\gamma$ in a 3-dimensional contact metric manifold $M$. The mean curvature vector field $H$ is said to be pseudo-Hermitian $C$-parallel if $\widehat{\nabla}_{T} \widehat{H}=\lambda \xi$. The vector field $H$ is said to be pseudo Hermitian C-proper mean curvature vector field if $\widehat{\triangle} \widehat{H}=$ $\lambda \xi$. Similarly, $H$ is said to be pseudo-Hermitian $C$-parallel vector field in the normal bundle if $\widehat{\nabla} \frac{\perp}{T} \widehat{H}=\lambda \xi$, and $H$ is said to be pseudo-Hermitian C-proper mean curvature vector field in the normal bundle if $\widehat{\triangle}^{\perp} \widehat{H}=\lambda \xi$, where $\lambda$ is a differentiable function along the curve.

Lemma 4.2. [14] Let $\gamma$ be a curve in a 3-dimensional contact metric manifold $M$. Then

$$
\begin{array}{r}
\widehat{\nabla}_{T} \widehat{\nabla}_{T} \widehat{\nabla}_{T} T=-3 \widehat{\kappa}^{\prime} T+\left(\widehat{\kappa}^{\prime \prime}-\widehat{\kappa}^{3}-\widehat{\kappa} \widehat{\tau}^{2}\right) N+\left(2 \widehat{\kappa}^{\prime} \widehat{\tau}+\widehat{\kappa} \widehat{\tau}^{\prime}\right) B \\
\widehat{\nabla}_{T}^{\perp} \widehat{\nabla}_{T}^{\perp} \widehat{\nabla}_{T}^{\perp} T=\left(\widehat{\kappa}^{\prime \prime}-\widehat{\kappa} \widehat{\tau}^{2}\right) N+\left(2 \widehat{\kappa}^{\prime} \widehat{\tau}+\widehat{\kappa} \widehat{\tau}^{\prime}\right) B
\end{array}
$$

and

$$
\begin{gathered}
\widehat{\triangle} \widehat{H}=-\widehat{\nabla}_{T} \widehat{\nabla}_{T} \widehat{\nabla}_{T} T, \\
\widehat{\triangle}^{\perp} \widehat{H}=-\widehat{\nabla}_{T}^{\perp} \widehat{\nabla}_{T}^{\perp} \widehat{\nabla}_{T}^{\perp} T .
\end{gathered}
$$

Using Lemma 4.2, we have the following theorem:

Theorem 4.3. A slant curve $\gamma$ in a 3-dimensional contact metric manifold $M$ has pseudo-Hermitian C-parallel mean curvature vector field if and only if it is a pseudo-Hermitian helix satisfying

$$
\widehat{\kappa}=\mp \sqrt{-\lambda \cos \alpha_{0}} \text { and } \widehat{\tau}=\mp \frac{\lambda \sin \alpha_{0}}{\sqrt{-\lambda \cos \alpha_{0}}},
$$

where $\lambda \cos \alpha_{0}<0$.

Proof. Assume that a slant curve $\gamma$ has pseudo-Hermitian $C$-parallel mean curvature vector field. Then from (4.2), the condition $\widehat{\nabla}_{T} \widehat{H}=\lambda \xi$ gives

$$
-\widehat{\kappa}^{2} T+\widehat{\kappa}^{\prime} N+\widehat{\kappa} \widehat{\tau} B=\lambda \xi .
$$

Since $\gamma$ is a slant curve we can write

$$
\xi=\cos \alpha_{0} T+\sin \alpha_{0} B .
$$

So using (4.7) and (4.8) we can write

$$
-\widehat{\kappa}^{2} T+\widehat{\kappa}^{\prime} N+\widehat{\kappa} \widehat{\tau} B=\lambda\left(\cos \alpha_{0} T+\sin \alpha_{0} B\right) .
$$

Taking the inner product of (4.9) with $N$ and using $\eta(N)=0$ we find $\widehat{\kappa}^{\prime}=0$, which implies that $\widehat{\kappa}$ is a constant. Hence from the equation (4.9), it follows that $\widehat{\kappa}=\mp \sqrt{-\lambda \cos \alpha_{0}}$. Since $\frac{\widehat{\tau}}{\hat{\kappa}}=\cot \alpha_{0}$ we obtain $\widehat{\tau}=\mp \frac{\lambda \sin \alpha_{0}}{\sqrt{-\lambda \cos \alpha_{0}}}$, where $\lambda \cos \alpha_{0}<0$.

The converse statement is trivial. 
Theorem 4.4. A slant curve $\gamma$ in a 3-dimensional contact metric manifold $M$ has pseudo-Hermitian $C$-parallel mean curvature vector field in the normal bundle if and only if it is a pseudo-Hermitian Legendre circle.

Proof. Assume that a slant curve $\gamma$ has pseudo-Hermitian $C$-parallel mean curvature vector field in the normal bundle. Then from (4.2), the condition $\widehat{\nabla} \frac{\perp}{T} \widehat{H}=\lambda \xi$ gives

$$
\widehat{\kappa}^{\prime} N+\widehat{\kappa} \widehat{\tau} B=\lambda\left(\cos \alpha_{0} T+\sin \alpha_{0} B\right) .
$$

So we have

$$
\begin{gathered}
\widehat{\kappa}^{\prime}=0, \\
\lambda \cos \alpha_{0}=0, \\
\widehat{\kappa} \widehat{\tau}=\lambda \sin \alpha_{0} .
\end{gathered}
$$

Then $\widehat{\kappa}$ is a constant. From (4.12), if $\cos \alpha_{0}=0$, then $\alpha_{0}=\pi / 2$. So it is a Legendre curve. Then from Proposition 3.4, $\widehat{\tau}=0$, which implies $\gamma$ is a pseudo-Hermitian Legendre circle. Moreover, from (4.13) we have $\lambda=0$.

The converse statement is trivial.

Theorem 4.5. There does not exist non-geodesic slant curve in a 3-dimensional contact metric manifold $M$ with pseudo Hermitian $C$-proper mean curvature.

Proof. Assume that $\gamma$ is a non-geodesic slant curve with contact angle $\alpha_{0}$ and has pseudo Hermitian $C$-proper mean curvature field. Then by definition, $\widehat{\triangle} \widehat{H}=\lambda \xi$. Using (4.6) and (4.8), we get

$$
\begin{gathered}
3 \widehat{\kappa} \widehat{\kappa}^{\prime} T-\left(\widehat{\kappa}^{\prime \prime}-\widehat{\kappa}^{3}-\widehat{\kappa} \widehat{\tau}^{2}\right) N-\left(2 \widehat{\kappa}^{\prime} \widehat{\tau}+\widehat{\kappa} \widehat{\tau}^{\prime}\right) B \\
=\lambda\left(\cos \alpha_{0} T+\sin \alpha_{0} B\right) .
\end{gathered}
$$

Hence we have

$$
\begin{gathered}
3 \widehat{\kappa} \widehat{\kappa}^{\prime}=\lambda \cos \alpha_{0}, \\
\widehat{\kappa}^{\prime \prime}-\widehat{\kappa}^{3}-\widehat{\kappa} \widehat{\tau}^{2}=0, \\
2 \widehat{\kappa}^{\prime} \widehat{\tau}+\widehat{\kappa} \widehat{\tau}^{\prime}=-\lambda \sin \alpha_{0} .
\end{gathered}
$$

So using $\frac{\widehat{T}}{\hat{\kappa}}=\cot \alpha_{0}$, we find $\lambda=0$. Then using Theorem 4.4. in [14], we find $\widehat{\kappa}=0$. Since $\gamma$ is not a geodesic, it can not have pseudo Hermitian $C$-proper mean curvature.

This completes the proof.

Theorem 4.6. A slant curve $\gamma$ in a 3-dimensional contact metric manifold $M$ has pseudo-Hermitian C-proper mean curvature field in the normal bundle if and only if either it is a Legendre curve with pseudo-Hermitian curvature $\widehat{\kappa}(s)=a s+b$, where $a$ and $b$ are real constants or it is a pseudo-Hermitian Legendre circle. 
Proof. Assume that $\gamma$ is a non-geodesic slant curve with contact angle $\alpha_{0}$ and has pseudo Hermitian $C$-proper mean curvature vector field in the normal bundle. Then by definition, $\widehat{\triangle}^{\perp} \widehat{H}=\lambda \xi$. Using (4.6) and (4.8), we get

$$
-\left(\widehat{\kappa}^{\prime \prime}-\widehat{\kappa} \widehat{\tau}^{2}\right) N-\left(2 \widehat{\kappa}^{\prime} \widehat{\tau}+\widehat{\kappa} \widehat{\tau}^{\prime}\right) B=\lambda\left(\cos \alpha_{0} T+\sin \alpha_{0} B\right) .
$$

Then we have

$$
\begin{aligned}
\widehat{\kappa}^{\prime \prime}-\widehat{\kappa} \widehat{\tau}^{2} & =0, \\
-\left(2 \widehat{\kappa}^{\prime} \widehat{\tau}+\widehat{\kappa} \widehat{\tau}^{\prime}\right) & =\lambda \sin \alpha_{0}, \\
\lambda \cos \alpha_{0} & =0 .
\end{aligned}
$$

From (4.17), if $\cos \alpha_{0}=0$, then $\alpha_{0}=\pi / 2$. So it is a Legendre curve. Then from Proposition 3.4, $\widehat{\tau}=0$. Thus the equations (4.15) and (4.16) give us $\lambda=0$. Then by Theorem 4.7 in [14], it follows that $\gamma$ is a Legendre curve with pseudo-Hermitian curvature $\widehat{\kappa}(s)=a s+b$, where $a$ and $b$ are real constants. If $\cos \alpha_{0} \neq 0$ and $\lambda=0$ then in view of Theorem 4.7 in [14], it follows that $\gamma$ is a pseudo-Hermitian Legendre circle.

The converse statement is trivial.

5

\section{Slant Curves of Sasakian Heisenberg Group with Pseudo-Hermitian CONNECTION}

The Heisenberg group $H_{3}$ can be viewed as $\mathbb{R}^{3}$ equipped with Riemannian metric

$$
g=d x^{2}+d y^{2}+\eta \otimes \eta,
$$

where $(x, y, z)$ are standard coordinates in $\mathbb{R}^{3}$ and

$$
\eta=d z+y d x-x d y .
$$

The 1-form $\eta$ satisfies $d \eta \wedge \eta=-\lambda d x \wedge d y \wedge d z$. Hence $\eta$ is a contact form. In [10], Inoguchi obtained the Levi-Civita connection $\nabla$ of the metric $g$ with respect to the left-invariant orthonormal basis

$$
e_{1}=\frac{\partial}{\partial x}-y \frac{\partial}{\partial z}, e_{2}=\frac{\partial}{\partial y}+x \frac{\partial}{\partial z}, e_{3}=\frac{\partial}{\partial z} .
$$

He obtained

$$
\begin{array}{ccc}
\nabla_{e_{1}} e_{1}=0, & \nabla_{e_{1}} e_{2}=e_{3}, & \nabla_{e_{1}} e_{3}=-e_{2} \\
\nabla_{e_{2}} e_{1}=-e_{3}, & \nabla_{e_{2}} e_{2}=0, & \nabla_{e_{2}} e_{3}=e_{1} \\
\nabla_{e_{3}} e_{1}=-e_{2}, & \nabla_{e_{3}} e_{2}=e_{1}, & \nabla_{e_{3}} e_{3}=0
\end{array}
$$

We also have the Heisenberg brackets

$$
\left[e_{1}, e_{2}\right]=2 e_{3}, \quad\left[e_{2}, e_{3}\right]=\left[e_{3}, e_{1}\right]=0 .
$$


Let $\varphi$ be the (1,1)-tensor field defined by $\varphi\left(e_{1}\right)=e_{2}, \varphi\left(e_{2}\right)=-e_{1}$ and $\varphi\left(e_{3}\right)=0$. Then using the linearity of $\varphi$ and $g$ we have

$$
\eta\left(e_{3}\right)=1, \quad \varphi^{2}(X)=-X+\eta(X) e_{3}, \quad g(\varphi X, \varphi Y)=g(X, Y)-\eta(X) \eta(Y) .
$$

We also have

$$
d \eta(X, Y)=g(X, \varphi Y)
$$

for all $X, Y \in \chi(M)$. Thus for $\xi=e_{3},(\varphi, \xi, \eta, g)$ is a contact metric structure and the Heisenberg group $H_{3}$ is a Sasakian space form of constant holomorphic sectional curvature $-3[10]$.

Now, let $\gamma: I \rightarrow H_{3}$ be a slant curve with contact angle $\alpha_{0}$. Assume that $\gamma$ is parametrized by arc length $s$ and $\{T, N, B\}$ denote the Frenet frame of $\gamma$. Then we can write

$$
T=\sin \alpha_{0} \cos \beta e_{1}+\sin \alpha_{0} \sin \beta e_{2}+\cos \alpha_{0} e_{3},
$$

where $\beta=\beta(s)$. Using (5.1) we have

$$
\begin{gathered}
\nabla_{T} T=\left(-\sin \alpha_{0} \sin \beta\left(\beta^{\prime}-2 \cos \alpha_{0}\right)\right) e_{1} \\
+\left(\sin \alpha_{0} \cos \beta\left(\beta^{\prime}-2 \cos \alpha_{0}\right)\right) e_{2} .
\end{gathered}
$$

On the other hand by the use of (5.2) it follows that

$$
\varphi T=-\sin \alpha_{0} \sin \beta e_{1}+\sin \alpha_{0} \cos \beta e_{2} .
$$

By the use of (2.7) we find

$$
\widehat{\nabla}_{T} T=-\beta^{\prime} \sin \alpha_{0} \sin \beta e_{1}+\beta^{\prime} \sin \alpha_{0} \cos \beta e_{2} .
$$

Since $\widehat{\nabla}_{T} T=\widehat{\kappa} N$, the equation (5.5) gives us

$$
\widehat{\kappa}=\left|\beta^{\prime}\right| \sin \alpha_{0} .
$$

Hence the principal normal vector field $N$ of $\gamma$ can be written as

$$
N=\operatorname{sgn}\left(\beta^{\prime}\right)\left(-\sin \beta e_{1}+\cos \beta e_{2}\right) .
$$

Since $B=T \times N$, we find

$$
B=\operatorname{sgn}\left(\beta^{\prime}\right)\left(-\cos \alpha_{0} \cos \beta e_{1}-\cos \alpha_{0} \sin \beta e_{2}+\sin \alpha_{0} e_{3}\right) .
$$

Then it is easy to see that

$$
B^{\prime}=\operatorname{sgn}\left(\beta^{\prime}\right)\left(\beta^{\prime} \cos \alpha_{0}-\cos 2 \alpha_{0}\right)\left(\sin \beta e_{1}-\cos \beta e_{2}\right),
$$

which gives us

$$
\widehat{\tau}=\beta^{\prime} \cos \alpha_{0}-\cos 2 \alpha_{0} .
$$


Now assume that $\widehat{\tau}=0$. Then from Proposition 3.4, $\gamma$ is a Legendre curve. Using (5.7), we obtain

$$
\beta(s)=\frac{\cos 2 \alpha_{0}}{\cos \alpha_{0}} s+c,
$$

where $c$ is a real constant. Hence from (5.6), $\widehat{\kappa}$ is a constant.

Let $\gamma(s)=(x(s), y(s), z(s))$. To find the explicit equations, we should integrate the system $\frac{d \gamma}{d s}=T$. Then

$$
\begin{gathered}
\frac{d x}{d s}=\sin \alpha_{0} \cos \left(\frac{\cos 2 \alpha_{0}}{\cos \alpha_{0}} s+c\right), \\
\frac{d y}{d s}=\sin \alpha_{0} \sin \left(\frac{\cos 2 \alpha_{0}}{\cos \alpha_{0}} s+c\right), \\
\frac{d z}{d s}=\cos \alpha_{0}+\frac{1}{2} \sin \alpha_{0}\left(\sin \left(\frac{\cos 2 \alpha_{0}}{\cos \alpha_{0}} s+c\right) x(s)-\cos \left(\frac{\cos 2 \alpha_{0}}{\cos \alpha_{0}} s+c\right) y(s)\right) .
\end{gathered}
$$

So using the method given in [4], the integration of above system gives the following example:

Example 5.1. Let $\gamma: I \rightarrow H_{3}$ be a curve with the following parametric equations.

$$
\begin{gathered}
x(s)=\frac{\cos \alpha_{0}}{\cos 2 \alpha_{0}} \sin \alpha_{0} \sin \left(\frac{\cos 2 \alpha_{0}}{\cos \alpha_{0}} s+c\right)+d_{1}, \\
y(s)=-\frac{\cos \alpha_{0}}{\cos 2 \alpha_{0}} \sin \alpha_{0} \cos \left(\frac{\cos 2 \alpha_{0}}{\cos \alpha_{0}} s+c\right)+d_{2}, \\
z(s)=\left(\cos \alpha_{0}+\frac{\cos \alpha_{0}}{\cos 2 \alpha_{0}} \sin ^{2} \alpha_{0}\right) s-d_{1} \frac{\cos \alpha_{0}}{\cos 2 \alpha_{0}} \sin \alpha_{0} \cos \left(\frac{\cos 2 \alpha_{0}}{\cos \alpha_{0}} s+c\right) \\
-d_{2} \frac{\cos \alpha_{0}}{\cos 2 \alpha_{0}} \sin \alpha_{0} \sin \left(\frac{\cos 2 \alpha_{0}}{\cos \alpha_{0}} s+c\right)+d_{3} .
\end{gathered}
$$

Then $\gamma$ is a pseudo-Hermitian Legendre circle with pseudo-Hermitian curvature $\widehat{\kappa}=\left|\frac{\cos 2 \alpha_{0}}{\cos \alpha_{0}}\right| \sin \alpha_{0}$, where $c, d_{1}, d_{2}$ and $d_{3}$ are some real constants.

Now assume that $\widehat{\tau} \neq 0$ and $\widehat{\kappa}$ is a constant. Then from (5.6), $\beta^{\prime}$ is a constant. Then we can write $\beta(s)=a s+b$, where $a$ and $b$ are real constants. By the use of equation (5.7), we find $\widehat{\tau}=a \cos \alpha_{0}-\cos 2 \alpha_{0}$. Hence $\widehat{\tau}$ is a constant. Similar to the method using in previous example, let $\gamma(s)=(x(s), y(s), z(s))$. To find the explicit equations, we should integrate the system $\frac{d \gamma}{d s}=T$. Then

$$
\begin{gathered}
\frac{d x}{d s}=\sin \alpha_{0} \cos (a s+b), \\
\frac{d y}{d s}=\sin \alpha_{0} \sin (a s+b), \\
\frac{d z}{d s}=\cos \alpha_{0}+\frac{1}{2} \sin \alpha_{0}(\sin (a s+b) x(s)-\cos (a s+b) y(s)) .
\end{gathered}
$$

Similarly, using the method given in [4], the integration of above system gives the following example: 
Example 5.2. Let $\gamma: I \rightarrow H_{3}$ be a curve with the following parametric equations.

$$
\begin{gathered}
x(s)=\frac{1}{a} \sin \alpha_{0} \sin (a s+b)+c_{1}, \\
y(s)=-\frac{1}{a} \sin \alpha_{0} \cos (a s+b)+c_{2}, \\
z(s)=\left(\cos \alpha_{0}+\frac{1}{a} \sin ^{2} \alpha_{0}\right) s-\frac{c_{1}}{a} \sin \alpha_{0} \cos (a s+b) \\
-\frac{c_{2}}{a} \sin \alpha_{0} \sin (a s+b)+c_{3} .
\end{gathered}
$$

Then $\gamma$ is a pseudo-Hermitian slant helix with pseudo-Hermitian curvature $\widehat{\kappa}=$ $|a| \sin \alpha_{0}$ and pseudo-Hermitian torsion $\widehat{\tau}=a \cos \alpha_{0}-\cos 2 \alpha_{0}$, where $a, b, c_{1}, c_{2}$ and $c_{3}$ are some real constants such that $a \neq \frac{\cos 2 \alpha_{0}}{\cos \alpha_{0}}$.

\section{REFERENCES}

[1] Arroyo, J., Barros, M., Garay, O. J., A characterisation of helices and Cornu spirals in real space forms, Bull. Austral. Math. Soc. 56 (1997), $37-49$.

[2] Barros, M., Garay, O. J., On submanifolds with harmonic mean curvature, Proc. Amer. Math. Soc. 123 (1995), 2545-2549.

[3] Blair, D. E., Riemannian geometry of contact and symplectic manifolds, second edition. Progress in Mathematics, 203. Birkhäuser Boston, Inc., Boston, MA, 2010.

[4] Caddeo, R., Oniciuc, C., Piu, P., Explicit formulas for non-geodesic biharmonic curves of the Heisenberg group, Rend. Sem. Mat. Univ. Politec. Torino 62 (2004), 265-277.

[5] Călin, C., Crasmareanu, M., Slant curves in 3-dimensional normal almost contact geometry, Mediterr. J. Math. 10 (2013), 1067-1077.

[6] Călin, C., Crasmareanu, M., Slant and Legendre curves in Bianchi-CartanVranceanu geometry, Czechoslovak Math. J. 64(139) (2014), 945-960.

[7] Chen, B.-Y., Some open problems and conjectures on submanifolds of finite type, Soochow J. Math. 17 (1991), 169-188.

[8] Cho, J. T., Lee, J.-E., Slant curves in contact pseudo-Hermitian 3manifolds, Bull. Aust. Math. Soc. 78 (2008), 383-396.

[9] Cho, J. T., Inoguchi, J., Lee, J.-E., On slant curves in Sasakian 3manifolds, Bull. Austral. Math. Soc. 74 (2006), 359-367. 
[10] Inoguchi, J., Minimal surfaces in the 3-dimensional Heisenberg group, Differ. Geom. Dyn. Syst. 10 (2008), 163-169.

[11] Inoguchi, J., Lee, J.-E., Slant curves in 3-dimensional almost contact metric geometry, Int. Electron. J. Geom. 8 (2015), 106-146.

[12] Lee, J.-E., On Legendre curves in contact pseudo-Hermitian 3-manifolds, Bull. Aust. Math. Soc. 81 (2010), 156-164.

[13] Lee, J.-E., Suh, Y. J., Lee, H., C-parallel mean curvature vector fields along slant curves in Sasakian 3-manifolds, Kyungpook Math. J. 52 (2012), 4959.

[14] Özgür, C., Güvenç, Ş., On some types of slant curves in contact pseudoHermitian 3-manifolds, Ann. Polon. Math. 104 (2012), 217-228.

[15] Güvenç, Ş., Özgür, C., On slant curves in trans-Sasakian manifolds, Rev. Un. Mat. Argentina 55 (2014), 81-100.

[16] Tanaka, N., On non-degenerate real hypersurfaces, graded Lie algebras and Cartan connections, Japan. J. Math. (N.S.) 2 (1976), 131-190.

[17] Tanno, S., Variational problems on contact Riemannian manifolds, Trans. Amer. Math. Soc. 314 (1989), 349-379.

[18] Webster, S. M., Pseudo-Hermitian structures on a real hypersurface, J. Diff. Geom. 13 (1978), 25-41. 